\title{
Bunch length compression manipulations
}

\author{
K. M. Fung, ${ }^{1}$ M. Ball, ${ }^{1}$ C. M. Chu, ${ }^{1}$ B. Hamilton,,${ }^{1}$ S. Y. Lee, ${ }^{1}$ and K. Y. $\mathrm{Ng}^{2}$ \\ ${ }^{1}$ Department of Physics, Indiana University, Bloomington, Indiana 47405 \\ ${ }^{2}$ Fermilab, Batavia, Illinois 60510
}

(Received 18 July 2000; published 2 October 2000)

\begin{abstract}
Bunch length compression of a single proton bunch is studied by manipulations of the rf voltage and phase. Analytical expressions for the compression ratio, defined as the ratio of the initial to the final bunch lengths, are derived for a linear model. Results obtained from numerical simulations are compared with experimental results. The ultimate maximum compression ratios are found to be $\sqrt{2} / \sqrt{3} \sigma_{\phi, i}$ for the phase shift method and $\sqrt{8} / \sqrt{3 \pi} \sigma_{\phi, i}$ for the voltage manipulation method, where $\sigma_{\phi, i}$ is the rms rf-phase spread of the initial beam bunch at the maximum rf voltage. Effects of space charge force are also studied.
\end{abstract}

PACS numbers: 29.27.Eg, 41.85.Ew

\section{INTRODUCTION}

Techniques in bunch beam manipulation are important for attaining high quality beams in accelerators and storage rings. In particular, bunch length shortening plays an important role in achieving high capture efficiency for the secondary beam (such as antiprotons, kaons, pions, muons, etc.) production, in emittance matching during a bucket to bucket transfer, etc.

In synchrotron and storage rings, the bunch length compression of beams can be attained by the manipulation of rf voltage and rf phase. This paper studies various experimental procedures in bunch rotation of space charge dominated beams and their dependence on the operational parameters of the accelerators and beams. We organize our paper as follows. Beam properties and experimental procedures in the Indiana University Cyclotron Facility (IUCF) cooler ring are discussed in Sec. II. Results of our rf voltage manipulation experiments and numerical analyses are presented in Sec. III. Results of our rf phase manipulation are compared with theory and numerical simulations in Sec. IV. Effects of space charge force on our bunch rotation are discussed in Sec. V. The conclusion is presented in Sec. VI.

\section{BEAM PROPERTIES AT THE IUCF COOLER RING}

The IUCF cooler is a six-sided storage ring with electron cooling [1]. The circumference is $86.82 \mathrm{~m}$ with proton beam injected from the cooler injector synchrotron (CIS) with a circumference $1 / 5$ of that of the cooler [2]. Our experiments were carried out in the cooler, where the betatron tunes were $\nu_{x}=3.805$ and $\nu_{z}=4.82$ so that nonlinear betatron resonances were not important to the circulating beams. The Princeton-Pennsylvania Accelerator (PPA) rf cavity was operating at $h=5$ harmonic. The basic parameters for our experiment are listed in Table I.

One proton bunch with intensity of several hundred $\mu \mathrm{A}$ was injected to the IUCF cooler and the longitudinal beam profile was measured as a function of time while the rf voltage or phase was being manipulated. The time evolution of the longitudinal beam profile was digitized at a rate of $12 \mathrm{kHz}$ in synchronization with the rf cavity frequency. The interval between consecutive beam profile is $83.33 \mu \mathrm{s}$, while each data set consists of 500 profiles and therefore has a time span of about $42 \mathrm{~ms}$. Each profile is digitized at 2 ns per channel and it has 502 channels corresponding to a time span of $1.004 \mu \mathrm{s}$.

Figure 1 shows a typical longitudinal beam profile $\rho(\tau)$ digitized in 2 ns time step in the IUCF cooler. The centroid and rms bunch length of the beam can be obtained from the integrals

$$
\begin{gathered}
N_{\mathrm{B}}=\int \rho(\tau) d \tau, \\
\bar{\tau}=\frac{1}{N_{\mathrm{B}}} \int \tau \rho(\tau) d \tau, \\
\sigma_{\tau}^{2}=\frac{1}{N_{\mathrm{B}}} \int(\tau-\bar{\tau})^{2} \rho(\tau) d \tau,
\end{gathered}
$$

where $N_{\mathrm{B}}$ is the number of particles in a bunch. Since the bunch profile was measured in synchronization with the rf cavity frequency, there existed a time coordinate $\tau_{s}$ that was synchronized with the $\mathrm{rf}$ wave, and the variation of $\bar{\tau}-\tau_{s}$ is small. In fact, the time dependence of $\bar{\tau}-\tau_{s}$ measures the dipole mode of the beam. Figure 2 shows an

TABLE I. A list of parameters for the experiment.

\begin{tabular}{lcc}
\hline \hline \multicolumn{1}{c}{ Parameter } & Symbol & Value \\
\hline Harmonic number & $h$ & 5 \\
Circumference & $C$ & $86.826 \mathrm{~m}$ \\
Momentum compaction factor & $\alpha$ & 0.0473 \\
Kinetic energy & $K E$ & $202.8 \mathrm{MeV}$ \\
Revolution frequency & $f_{0}$ & $1.96632 \mathrm{MHz}$ \\
rf frequency & $f_{\mathrm{rf}}$ & $9.8316 \mathrm{MHz}$ \\
Phase slip factor & $\eta$ & -0.6288 \\
Synchrotron tune at $V_{\mathrm{rf}}=1000 \mathrm{~V}$ & $\nu_{s}$ & $1.164 \times 10^{-3}$ \\
\hline \hline
\end{tabular}




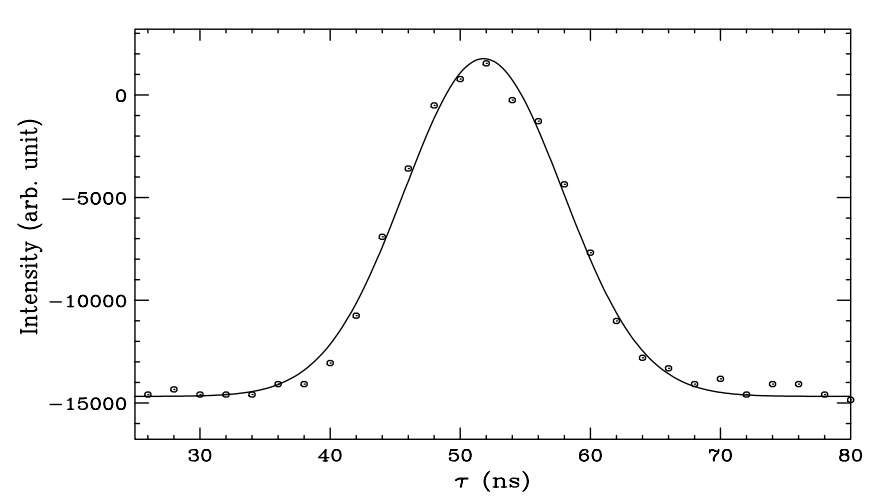

FIG. 1. A typical beam profile of cooled beam at the IUCF cooler ring. The profile can be fitted reasonably well by a Gaussian distribution shown as the solid line.

example of beam profile evolution during the bunch rotation through rf voltage manipulation. Note that the beam profile during the bunch rotation deviates from Gaussian shape. The rms value derived depends very much on the tail distribution. Thus we often resort our analysis to the full width at half maximum (FWHM) method to derive the bunch length. Furthermore, the rms bunch length is very sensitive to the background cut of the beam distribution, while the FWHM is less sensitive to the background cut. Because we turned off the phase feedback loop during our experiments, the beam loading on the rf system might induce a small dipole mode oscillation that may, in turn, cause asymmetry in the beam distribution shown in Fig. 2.

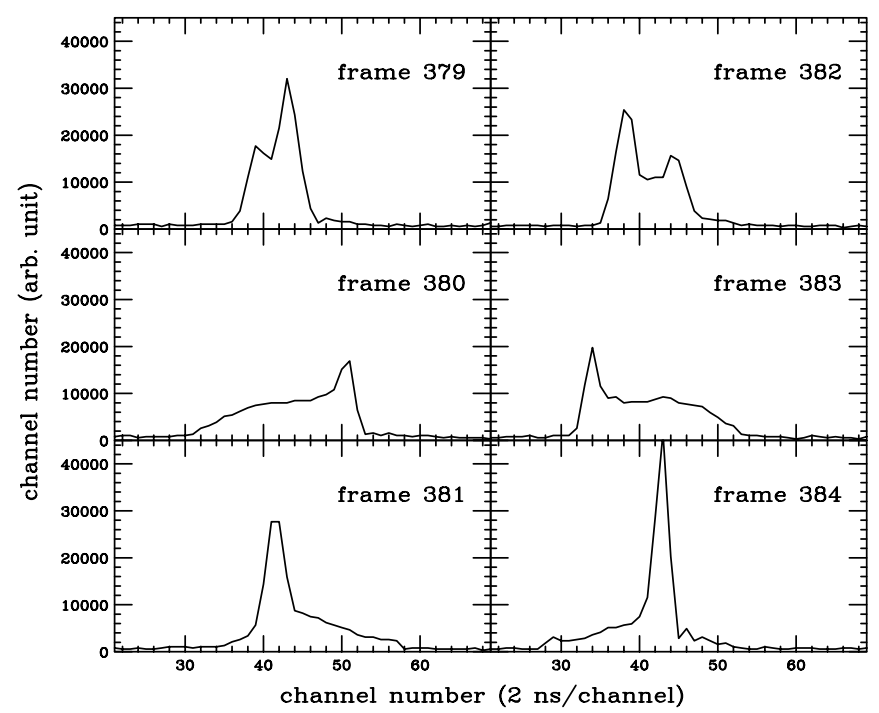

FIG. 2. The evolution of a beam profile after the voltage is jumped to a high value. Each frame is separated by about $83.33 \mu \mathrm{s}$ or 164 revolutions. Parameters for this run are $I_{\text {avg }}=670 \mu \mathrm{A}$, rf voltage ramp-down time from $V_{0}=350 \mathrm{~V}$ to $V_{1}=5 \mathrm{~V}$ is $27 \mathrm{~ms}$, and the rf voltage is suddenly raised to $V_{2}=1000 \mathrm{~V}$ in about 20 turns. The asymmetry in each beam profile indicated that there was a small dipole mode, i.e., $\bar{\tau}-\tau_{s} \neq 0$. The dipole mode may arise from the beam loading on the rf system.
With the synchrotron phase space coordinates $\phi=-2 \pi f_{\mathrm{rf}}\left(\tau-\tau_{\mathrm{s}}\right)$ as the synchrotron phase coordinate relative to the synchronous phase and $\delta=\Delta p / p_{0}$ as the fractional off-momentum coordinate, the synchrotron mapping equation is [3]

$$
\begin{gathered}
\phi_{n+1}=\phi_{n}+2 \pi h \eta \delta_{n}, \\
\delta_{n+1}=\delta_{n}+\frac{e V_{\mathrm{rf}}}{\beta^{2} E} \sin \phi_{n+1},
\end{gathered}
$$

where $\phi_{n}$ and $\delta_{n}$ are the phase space coordinates of a particle at the $n$th turn, $h$ is the harmonic number, $V_{\mathrm{rf}}$ is the rf voltage, $\beta c$ is the speed of the orbiting particle, $\eta$ is the phase slip factor, $E$ is the beam energy, and the synchronous phase angle is $\phi_{s}=0$. Since it takes thousands of revolutions to complete one synchrotron oscillation, the mapping equations can be represented by differential equations, and the motion is described by a synchrotron Hamiltonian

$$
H=\frac{1}{2} h \eta \delta^{2}+\frac{e V_{\mathrm{rf}}}{2 \pi \beta^{2} E}(\cos \phi-1),
$$

where the independent variable is the orbiting angle $\theta$, which advances by $2 \pi$ per revolution.

During the storage mode, proton beams in the IUCF cooler ring are cooled to the center of phase space by an electron cooling system, where a dc electron beam at low temperature is brought to exchange energy with the circulating protons. The cooling time is of the order of $0.3 \mathrm{~s}$ [4]. Beam particles in an accelerator experience diffusion processes such as beam-gas scattering, intrabeam Coulomb scattering, rf noise, power supply ripple, nonlinear resonances, etc. The equilibrium phase-space distribution function of a stochastic-damping system is given by [5]

$$
\tilde{\rho}(\phi, \delta)=\frac{N_{\mathrm{B}}}{\mathcal{N}} \exp \left(H / E_{\mathrm{th}}\right),
$$

where $\mathcal{N}$ is the normalization constant and $E_{\text {th }}$ is the longitudinal thermal energy. If the emittance of the cooled beam is small relative to the bucket area, the distribution function is approximately Gaussian evidently shown in Fig. 1 [6].

In small bunch approximation, the rms bunch area (in $\mathrm{eVs}$ ) is given by

$$
\mathcal{A}_{\mathrm{rms}}=\pi \sigma_{\tau} \sigma_{\Delta E}=\frac{\pi \beta^{2} E}{h \omega_{0}} \sigma_{\phi} \sigma_{\delta} \approx \frac{\pi \beta^{2} E \nu_{\mathrm{s}}}{h^{2}|\eta| \omega_{0}} \sigma_{\phi}^{2},
$$

where $\nu_{\mathrm{s}}=\sqrt{h|\eta| e V_{\mathrm{rf}} / 2 \pi \beta^{2} E}$ is the small amplitude synchrotron tune, $\sigma_{\phi}=h \omega_{0} \sigma_{\tau}$ is the rms beam width in the rf phase coordinate, and $\omega_{0}$ is the angular revolution frequency. The thermal energy is related to the rms bunch area by

$$
E_{\mathrm{th}}=\frac{h \omega_{0} \nu_{\mathrm{s}}}{\pi \beta^{2} E} \mathcal{A}_{\mathrm{rms}}
$$




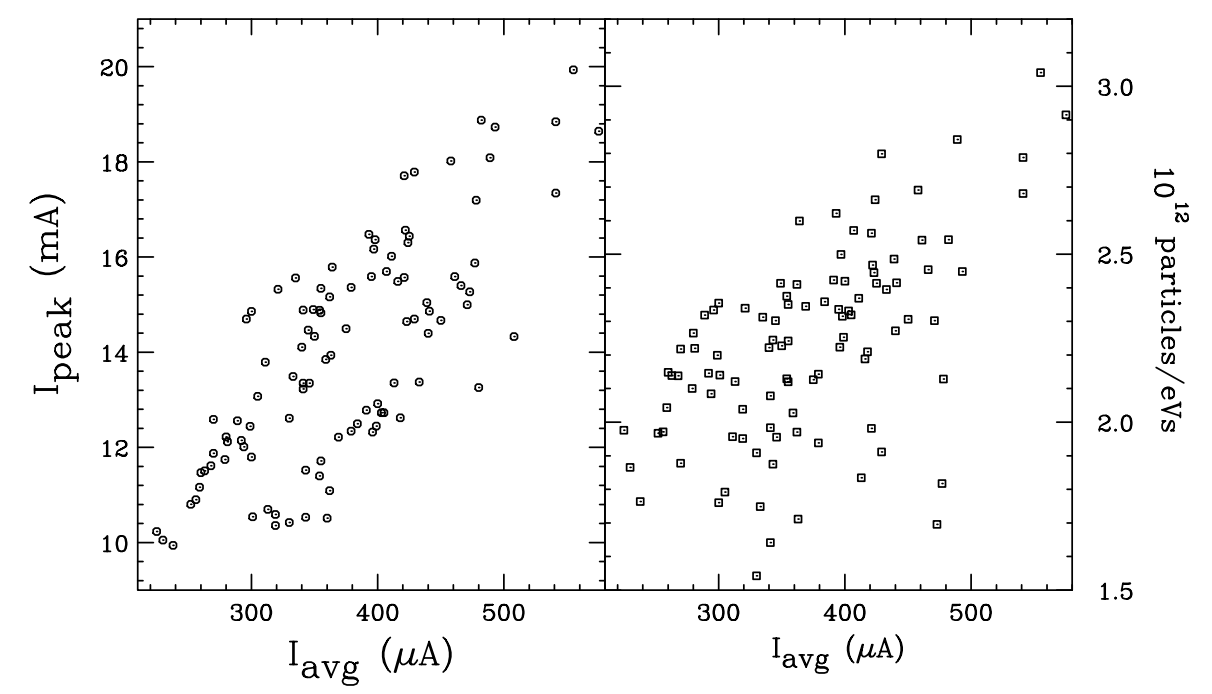

FIG. 3. Left: The peak beam intensity (in $\mathrm{mA}$ ) versus the average beam intensity (in $\mu \mathrm{A}$ ) for our phase-shift experimental data. Right: The beam brightness defined as the number of particles in a bunch divided by the rms phase space area. The unit is $10^{12}$ particles/eV s.

The peak current $I_{\text {peak }}$ and the beam brightness $\mathcal{B}$ are defined by

$$
I_{\text {peak }}=\frac{N_{\mathrm{B}} e}{\sqrt{2 \pi} \sigma_{\tau}}, \quad \mathcal{B}=\frac{N_{\mathrm{B}}}{\mathcal{A}_{\mathrm{rms}}} .
$$

Figure 3 shows, respectively, the peak current (left) and beam brightness (right) versus the average current. The spread in the experimental data reflects different injection conditions, electron-cooling setting, and other uncontrollable noises. On the other hand, Fig. 4 shows a compilation of the initial rms bunch length versus the rf voltage for all of our experimental data. The line corresponds to a simple formula of $28.1 / V_{\mathrm{rf}}^{1 / 4} \mathrm{~ns}$, where $V_{\mathrm{rf}}$ is in volts. There is considerable spread in the bunch length at a given rf voltage due to different beam currents, rf noise, etc. However, it seems that the initial phase space areas for most of our experimental conditions are almost equal to $\mathcal{A}_{\mathrm{rms}} \approx 6.6 \times 10^{-4} \mathrm{eV} \mathrm{s}$.

Bunch length compression often employs rf voltage and phase manipulations. The rms (or FWHM) bunch length can be derived during the dynamical evolution of bunch rotation. In particular, the compression ratio, defined as the ratio of the initial to final bunch lengths

$$
r_{c}=\sigma_{\tau, i} / \sigma_{\tau, f} \quad \text { or } \quad r_{c}=\tau_{\mathrm{FWHM}, i} / \tau_{\mathrm{FWHM}, f}
$$

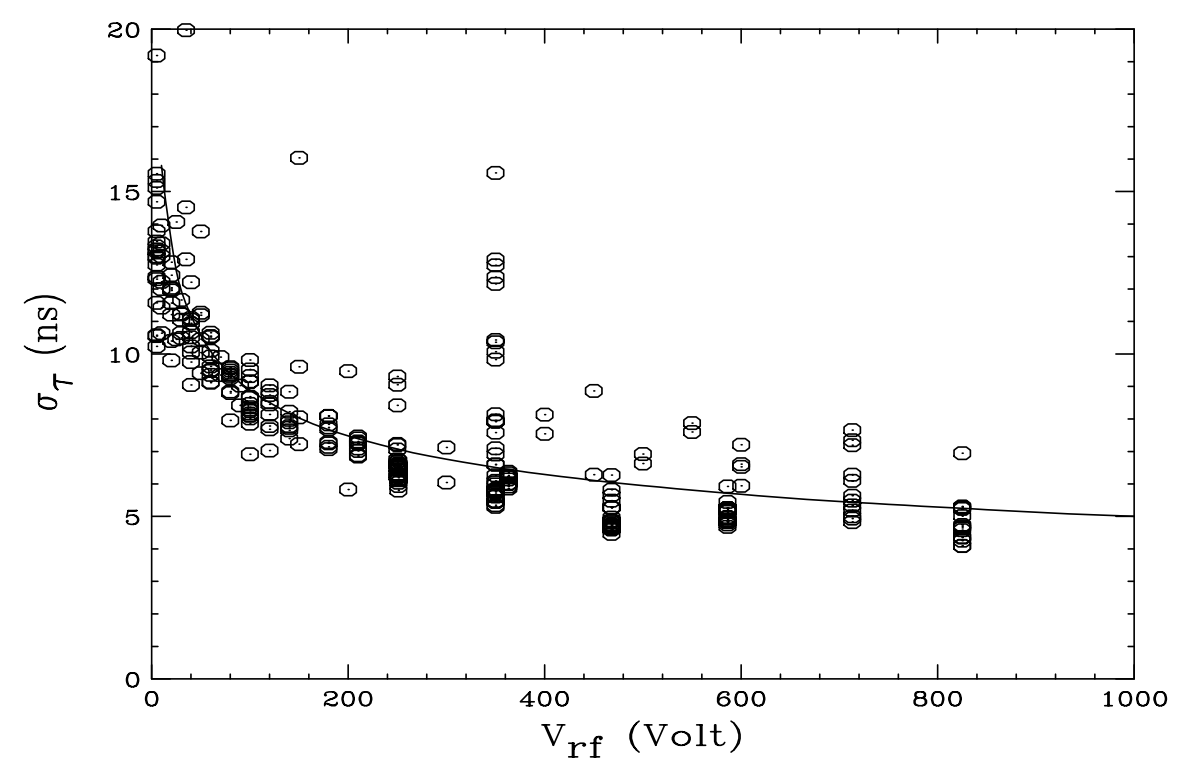

FIG. 4. Compilation of rms bunch length versus initial rf voltage of a cooled beam in the IUCF cooler ring. The line corresponds to a fit with $\sigma_{\tau}=28.1 / V_{\mathrm{rf}}^{1 / 4} \mathrm{~ns}$, where $V_{\mathrm{rf}}$ is measured in volts. 
can be obtained from the experimental data. We discuss the results of rf voltage and phase manipulations below. A fair comparison of two methods will be discussed in Sec. VI.

\section{BUNCH COMPRESSION BY RF VOLTAGE MANIPULATION}

\section{A. Results of rf voltage manipulation experiment}

First, we report our experiment of bunch length compression by using the rf voltage manipulation. The rf voltage was adiabatically decreased from $V_{\mathrm{rf}}=350 \mathrm{~V}$ to a low cavity voltage $V_{\text {rf }}=V_{1}$, then it was nonadiabatically increased to a high cavity voltage $V_{\mathrm{rf}}=V_{2}$. For the PPA cavity at the IUCF cooler ring, the achievable maximum rf cavity voltage is approximately $1000 \mathrm{~V}$. The duration for adiabatic process was chosen to be 5-30 ms. Since the synchrotron period at $V_{\mathrm{rf}}=5 \mathrm{~V}$ is about $6.2 \mathrm{~ms}$, beam loss will occur if the ramp-down time is too short. The time for the nonadiabatic voltage jump was about $10 \mu \mathrm{s}$, determined mainly by the response of power supply circuitry and the $Q$ value of rf cavity. Different values of $V_{1}$ and beam currents are used in order to study the achievable compression ratio. We should also note that the bucket area at $V_{\mathrm{rf}}=5 \mathrm{~V}$ is only 3.8 times the average rms bunch area of $6.6 \times 10^{-4} \mathrm{eV} \mathrm{s}$. Beam loss may occur for low $V_{1}$ values. Since the cooling time was very much longer than the rf voltage manipulation time, bunch area should remain conserved, as shown evidently in the low rf voltage region of Fig. 4.

As the voltage is raised nonadiabatically to $V_{2}$, the mismatched bunch begins to rotate. Figure 2 shows the longitudinal profiles digitized once in every $83.33 \mu$ s, which is a major hardware limitation in our experiment. At the $\mathrm{rf}$ voltage of $1000 \mathrm{~V}$, where the synchrotron tune is high, we digitize on average 5.24 frames in one synchrotron period. If we missed the peak of the first few synchrotron oscillations after the voltage jump, the beam bunch may filament and the measured final bunch length would be much larger.

Figure 5 shows the bunch length derived from FWHM (left) and rms (right) methods as a function of time. The bunch length is observed to increase in the first $30 \mathrm{~ms}$ when the rf voltage is adiabatically lowered [6]. As the rf voltage is nonadiabatically increased to $1000 \mathrm{~V}$, the bunch length begins to oscillate rapidly at twice the synchrotron frequency. If we zoom into the first few synchrotron oscillations after the rf voltage jump, we will find that we indeed missed the minimum bunch length of the first few synchrotron oscillations, but the bunch continued to perform rigid synchrotron motion after many synchrotron periods. A possible explanation is that the bucket area at $V_{\mathrm{rf}}=1000 \mathrm{~V}$ was about 8.9 times the bunch area, and thus the synchrotron motion of the bunch was essentially linear. Furthermore, the potential well distortion due to the space charge force may help to make the bunch rotation more rigid. Because the bunch shape is far from Gaussian during the bunch rotation stage, we will report our data based essentially on FWHM analysis.

The results of compression ratio based on FWHM analysis derived from our experiments are shown as crosses in Fig. 6, where $\sigma_{\tau, i}$ is the bunch length at $V_{\mathrm{rf}}=V_{1}$ and $\sigma_{\tau, f}$ is the minimum observable bunch length at $V_{\mathrm{rf}}=V_{2}$. For each voltage ratio $V_{2} / V_{1}$, there is a compression ratio spread because the initial beam condition of each data set is not identical. For instance, the initial rms bunch length $\sigma_{\tau, i}$ has a range from 6 to $20 \mathrm{~ns}$.

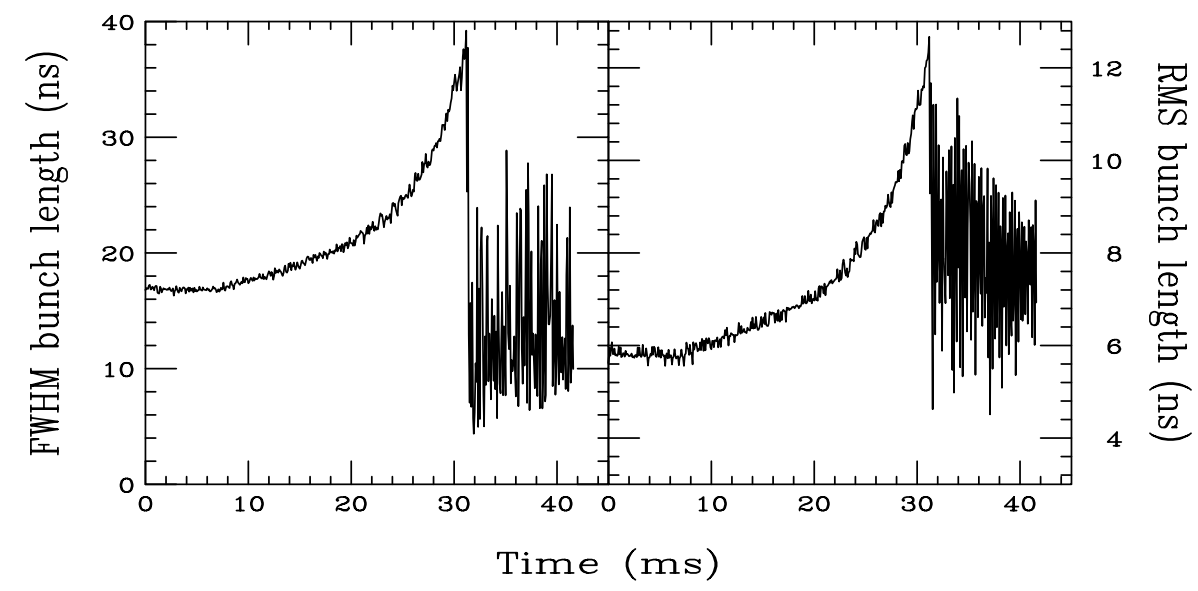

FIG. 5. The bunch length derived from FWHM (left) and rms (right) methods during the bunch compression using rf voltage manipulation. The beam condition is the same as that of Fig. 2. Since the profile during the bunch rotation stage deviates substantially from Gaussian, the bunch lengths derived from the FWHM method are much smaller than those derived from the rms method. The bunch length is seen to increase in the first $30 \mathrm{~ms}$, when the rf voltage is adiabatically lowered, and later oscillate rapidly with twice the synchrotron frequency after the rf voltage is nonadiabatically raised. 


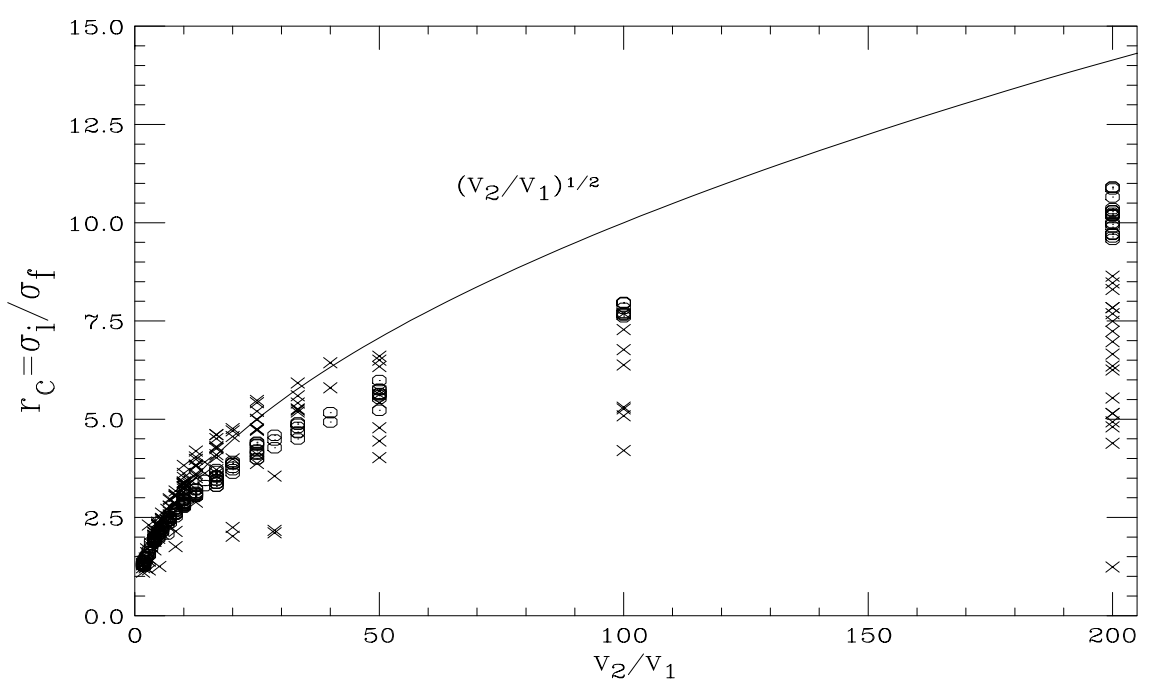

FIG. 6. Compression ratio versus voltage ratio. Experimental results are denoted by crosses. Simulation result is denoted by circles with initial condition according to the initial bunch length. Linear theory is denoted by the solid line. The value of $V_{2}$ is fixed at $1000 \mathrm{~V}$ while $V_{1}$ is varied to obtain different compression ratio. The compression ratio spread at each voltage ratio is caused by different initial beam conditions and experimental bandwidth limitation.

\section{B. A model with linear synchrotron motion}

First, we consider linear synchrotron motion. It is reasonable to assume that all nonadiabatic processes involved in the experiment are instantaneous since they have a time scale of $10 \mu \mathrm{s}$ that is much less than the synchrotron period of $1.38 \mathrm{~ms}$ at the $\mathrm{rf}$ voltage of $V_{1} \approx 100 \mathrm{~V}$. In small amplitude approximation, the rms bunch length and height $\sigma_{\tau, i}$ and $\sigma_{\delta, i}$ are [3]

$$
\begin{gathered}
\sigma_{\tau, i}=\sqrt{\frac{\mathcal{A}_{\mathrm{rms}}}{\omega_{0}}}\left(\frac{2|\eta|}{\pi h e V_{1} \beta^{2} E\left|\cos \phi_{s}\right|}\right)^{1 / 4}, \\
\sigma_{\delta, i}=\sqrt{\frac{\omega_{0} \mathcal{A}_{\mathrm{rms}}}{\pi \beta^{2} E}}\left(\frac{h e V_{1}\left|\cos \phi_{s}\right|}{2 \pi \beta^{2} E|\eta|}\right)^{1 / 4} .
\end{gathered}
$$

Here $\mathcal{A}_{\text {rms }}$ is the invariant rms phase space area in $\mathrm{eV} \mathrm{s}$. Typically $\mathcal{A}_{\text {rms }} \approx 6.6 \times 10^{-4} \mathrm{eV} \mathrm{s}$ for about $10^{9}$ particles. In the linearized model of synchrotron motion, the beam bunch performs rigid quadrupole mode oscillations when the rf voltage is nonadiabatically raised to $V_{2}$. At $1 / 4$ of the synchrotron period, the minimum rms bunch length becomes

$$
\sigma_{\tau, \mathrm{f}}=\sqrt{\frac{\mathcal{A}_{\mathrm{rms}}}{\omega_{0}}}\left(\frac{2|\eta|}{\pi \beta^{2} E h \mid \cos \phi_{s}}\right)^{1 / 4} \frac{\left(e V_{1}\right)^{1 / 4}}{\left(e V_{2}\right)^{1 / 2}} .
$$

Thus the compression ratio, in linear model, depends only on the rf voltage ratio; i.e.,

$$
r_{c}=\frac{\sigma_{\tau, i}}{\sigma_{\tau, f}}=\sqrt{\frac{V_{2}}{V_{1}}} .
$$

The solid line in Fig. 6 shows the expected compression ratio in linear model. At a maximum compression ratio, the phase space area of the beam fills up the entire bucket area for the rf voltage $V_{1}$. Thus the maximum compression ratio can be expressed as

$$
r_{c, \text { max }}=\frac{\mathcal{A}_{\mathrm{B}, \max }}{\mathcal{A}}
$$

where $\mathcal{A}_{\mathrm{B} \text {,max }}$ is the maximum bucket area of the accelerator rf system and $\mathcal{A}=6 \mathcal{A}_{\text {rms }}$ is the phase space area of $95 \%$ of the bunch.

\section{Numerical simulation and nonlinear model}

In reality, synchrotron motion is nonlinear. Using the synchrotron mapping equations, we track the evolution of the beam distribution. For each $\sigma_{\tau, i}$ obtained from experiment, the evolution of 10000 up to 80000 particles with an initial Gaussian distribution truncated at the bucket edge is followed. We obtain the bunch length $\sigma_{\tau}(t)$ each turn and hence the minimum bunch length $\sigma_{\tau, f}$ in the bunch rotation stage. The result is shown as circles in Fig. 6. The spread in the compression ratio arises essentially from the spread in the initial bunch length $\sigma_{\tau, i}$.

Because of nonlinear synchrotron tune spread, the resulting bunch compression ratio deviates substantially from $\sqrt{V_{2} / V_{1}}$ when $V_{2} / V_{1}$ is large. Thus it is difficult to achieve the maximum theoretical compression ratio Eq. (16). We also note that the compression ratios obtained from experimental measurements are usually below the numerical simulations.

\section{BUNCH COMPRESSION BY RF PHASE MANIPULATION}

\section{A. Experimental results}

In order to compress the beam width, the rf phase is nonadiabatically shifted by $\pi-2 \phi_{s}$ so that the beam 


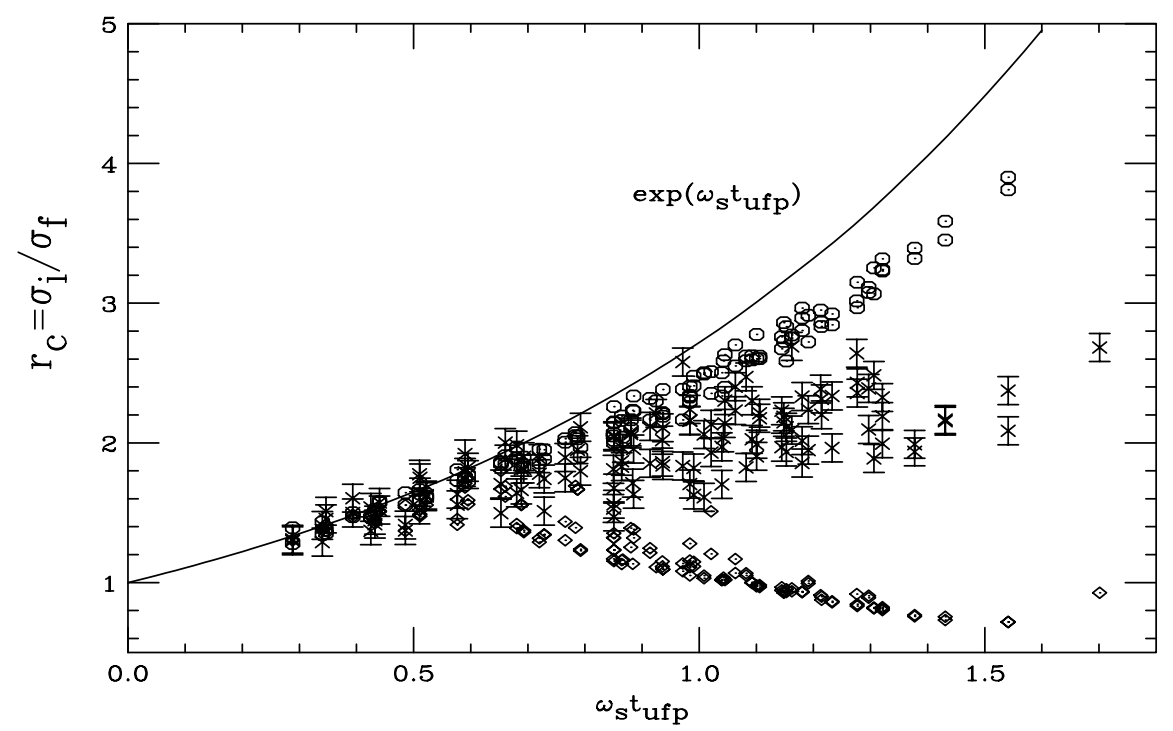

FIG. 7. Compression ratio versus $\omega_{s} t_{\mathrm{UFP}}$ where $\omega_{s}$ is the small amplitude synchrotron angular frequency and $t_{\mathrm{UFP}}$ is the time that the bunch stays at the UFP. The compression ratios derived from FWHM analysis of experimental data are denoted by crosses. Simulation results are denoted by circles using FWHM analysis and triangles with rms analysis. Linear theory is denoted by the solid line, i.e., $\exp \left\{\omega_{s} t_{\mathrm{UFP}}\right\}$. The compression ratio spread at each $\omega_{s} t_{\mathrm{UFP}}$ is caused by different initial beam conditions.

bunch sits at the unstable fixed point (UFP) $\pi-\phi_{s}$ of the $\mathrm{rf}$ bucket, where $\phi_{s}$ is the synchronous rf phase angle.

The motion of particle near UFP is linear and hyperboli$\mathrm{cal}$, the bunch area is preserved while the rf force compresses the bunch in one direction and stretches the bunch in another direction. After a time of $t_{\mathrm{UFP}}$, the rf phase is nonadiabatically restored, or the beam is kicked onto a transfer line. The bunch compression ratio is given by [3]

$$
\frac{\sigma_{\phi, i}}{\sigma_{\phi, f}}=\exp \left(\omega_{\mathrm{s}} t_{\mathrm{UFP}}\right)
$$

where $\sigma_{\phi, i}$ is the rms bunch length in rf phase just before the first phase shift, $\sigma_{\phi, f}$ is the minimum observable rms bunch length after the second phase shift, and $\omega_{s}=\omega_{0} \nu_{s}$ is the small amplitude synchrotron angular frequency.

Our experiment had been performed below transition with $\phi_{s}=0$. Each nonadiabatic rf phase jump takes about $10 \mu \mathrm{s}$, or about 20 revolutions, while the time stays at the UFP $t_{\mathrm{UFP}}$ is varied. The crosses in Fig. 7 show the observed bunch compression ratio versus $\omega_{s} t_{\mathrm{UFP}}$, where the solid line is the expected theoretical linear theory $\exp \left(\omega_{s} t_{\mathrm{UFP}}\right)$. Since the initial rms bunch length $\sigma_{\tau, i}$ has a range from 4 to $16 \mathrm{~ns}$ depending on the rf voltage and beam intensity, the measured compression ratio data also show a spread of compression ratio at each $\omega_{s} t_{\mathrm{UFP}}$. Note that when the compression ratio reaches a value of about 3 , in our experiment at the IUCF cooler, it also deviates substantially from the linear theory.

\section{B. Theoretical description and numerical simulations}

For particle simulation near UFP, we substitute the phase coordinate $\varphi=\phi-\pi$ in Eq. (5). Particle motion near $(\varphi, \delta)=(0,0)$ is hyperbolical. However, the total phase space area is preserved, thus the beam is stretched in one direction and compressed in another direction. The amount of compression depends on the time $t_{\mathrm{UFP}}$ that the bunch stays at the UFP. At a desired compression factor, the rf phase is shifted back to the stable fixed point (SFP) and the bunch starts to rotate. Figure 8 shows the phase space distribution of the initial Gaussian distribution (left), the phase space distribution after the rf phase is shifted back (middle), and the phase space distribution after $3 / 8$ of synchrotron period (right). Note here that the process of bunch stretching is very linear, as shown in the middle graph of Fig. 8 .

The bunch profile during the bunch rotation substantially deviates from Gaussian distribution (see the bottom-right plot of Fig. 2). Thus it is difficult to analyze numerical simulation data with rms or Gaussian fit. The diamond symbols in Fig. 7 show the compression ratio derived from the rms analysis of numerical simulations. The circles show the compression ratio obtained from the FWHM analysis of the same simulation data. The difference arises from the fact that the final bunch length differs from the Gaussian distribution. Experimental data obtained from the FWHM analysis are shown as crosses in Fig. 7.

We will now derive the ultimate bunch compression ratio for the rf phase shift method. In the normalized phase-space coordinate with $\mathcal{P}=-\left(h|\eta| / \nu_{s}\right)(\Delta p / p)$, the Hamiltonian for stationary synchrotron motion is transformed to (see p. 243 in Ref. [3])

$$
H_{0}=\frac{1}{2} \nu_{s} \mathcal{P}^{2}+2 \nu_{\mathrm{s}} \sin ^{2} \frac{\phi}{2},
$$

where $\nu_{s}$ is the small amplitude synchrotron tune, the orbital angle $\theta$ is the independent variable, and $(\phi, \mathcal{P})$ 


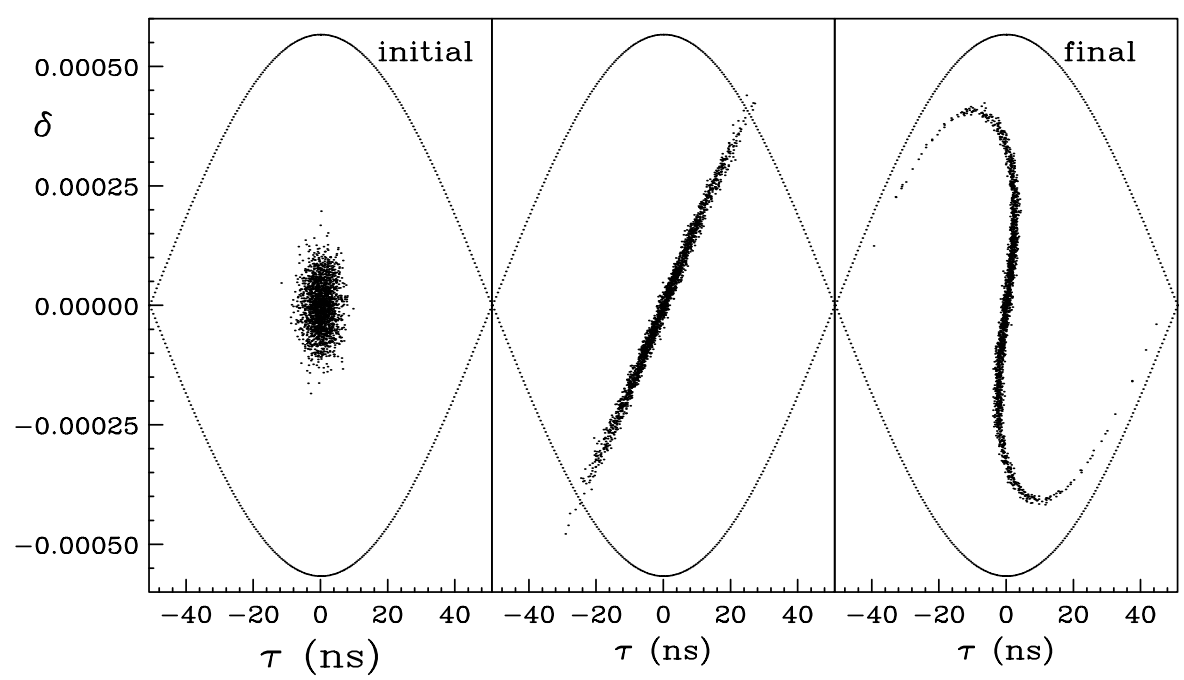

FIG. 8. Evolution of bunch population through rf phase jump. Note that the particle motion is relatively linear near the UFP. Nonlinear rf potential becomes most important when the rf phase is shifted back to the SFP.

are normalized conjugate phase-space coordinates. The Hamiltonian has a SFP at $(\phi, \mathcal{P})_{\mathrm{SFP}}=(0,0)$ and an UFP at $(\phi, \mathcal{P})_{\text {UFP }}=(\pi, 0)$. The synchrotron Hamiltonian is autonomous ( $\theta$ independent), and, thus, the Hamiltonian value is a constant of motion.

Near the UFP, the separatrix of the Hamiltonian in Eq. (18) can be approximated by two straight lines crossing at $45^{\circ}$ angles with the horizontal axis $\phi$. When the rf phase is shifted so that the beam sits on the UFP, the bunch width and height will stretch and compress along the separatrix. The rate of growth is equal to $\exp \left(\omega_{s} t_{\text {UFP }}\right)$ [3]. The maximum rf phase coordinate $\phi_{\max }$ that a bunch width can increase and still stay within the bucket after the $\mathrm{rf}$ phase is shifted back to SFP is given approximately by

$$
\frac{1}{2} \phi_{\max }^{2}+2 \sin ^{2}\left(\frac{\phi_{\max }}{2}\right) \approx 2
$$

where we assume linear approximation for particle motion near SFP. Thus we obtain $\phi_{\max } \approx \sqrt{2}$. Using Liouville's theorem, conservation of the phase space area, we find

$$
\pi \sigma_{\phi, i}^{2}=\pi \sigma_{\mathcal{P}, f} \sigma_{\phi, f} .
$$

Assuming that $95 \%$ of the beam particles reach $\phi_{\max }=$ $\sqrt{2}$ so that $\sigma_{\mathcal{P}, f}=(\sqrt{2} / \sqrt{6}) \phi_{\max }=\sqrt{2 / 3}$, we find the compression ratio as

$$
r_{c}=\frac{\sigma_{\phi, i}}{\sigma_{\phi, f}}=\frac{\sigma_{\mathcal{P}, f}}{\sigma_{\phi, i}} \approx \frac{\sqrt{2}}{\sqrt{3} \sigma_{\phi, \mathrm{i}}} .
$$

The time needed to reach this maximum compression ratio is

$$
\omega_{\mathrm{s}} \hat{t}_{\mathrm{UFP}}=\ln \frac{1}{\sigma_{\phi, i}}+\frac{1}{2} \ln \frac{2}{3} .
$$

Most of our experimental runs are in fact with $\phi_{\max }>\sqrt{2}$. When the rf phase is shifted back to the SFP, there has been beam loss and a much larger number of particles reside near the bucket edges than in the voltage manipulation experiment. Here the final phase rotation is $3 / 8$ of a synchrotron period, also much longer than that in the voltage manipulation experiment. As a result, longer tails with higher population are developed. This leads to much larger deviation from a Gaussian profile. This explains why the FWHM analysis is preferred over the rms analysis.

We did our phase shift experiments at rf voltages ranging from 250 to $825 \mathrm{~V}$. Because of the limitation of our digitizing hardware mentioned earlier, we digitize about 8.86 profiles in every synchrotron revolution period at an rf voltage of $350 \mathrm{~V}$. Although it is slightly easier to catch the minimum bunch length profile than our experimental observation with rf voltage manipulation, our results may still deviate from numerical simulations.

Another difficulty in our phase shift experiments is that the $\mathrm{rf}$ voltage remains at a relatively low rf voltage during and after the phase shift. The synchrotron motion during the bunch rotation stage would be much more nonlinear because the ratio of bucket area to the bunch area was smaller compared with that of the rf voltage manipulation experiments, where the rf voltage at the final stage of bunch rotation was $1000 \mathrm{~V}$.

The difficulty of nonlinear synchrotron motion in the final stage of bunch rotation can be solved by using the buncher in the transport line. After proper bunch compression, the beam is kicked out of the synchrotron and the $R_{56}$ transport matrix element will compress bunch; i.e., lower energy particles travel a shorter path and the higher energy particles travel a longer path. However, the resulting compression ratio is reduced by a factor of $1 / \sqrt{2}$. Since there is no constraint that the final bunch size should fit into the bucket, one can regain the factor of $\sqrt{2}$ by staying longer at the UFP.

\section{EFFECT OF SPACE CHARGE FORCE}

The wakefield induced by the space charge force can be described by a broadband impedance given by 


$$
\frac{Z_{\mathrm{spch}}^{\|}}{n}=-j \frac{g_{0} Z_{0}}{2 \beta \gamma^{2}} \approx-j 1.0 \times 10^{3} \Omega,
$$

where, in a uniform transverse distribution approximation, $g_{0}=\frac{1}{2}+2 \ln \frac{b}{a} \approx 4.5$ is the geometric factor and $Z_{0}=$ $\mu_{0} c=377 \Omega$ is the impedance of the vacuum. Two major effects arising from the space charge impedance are the potential well distortion and microwave instability.

\section{A. Potential well distortion}

The energy gain per revolution due to the space charge force is

$$
\Delta U_{\mathrm{spch}}=-\frac{e^{2}}{\omega_{0}}\left|\frac{Z_{\mathrm{spch}}^{\|}}{n}\right| \frac{\partial \rho}{\partial \tau} .
$$

Including the space charge force in numerical simulations, Eq. (5) should be modified as

$$
\delta_{n+1}=\delta_{n}+\frac{e V_{\mathrm{rf}}}{\beta^{2} E} \sin \phi_{n+1}+\frac{\Delta U_{\mathrm{spch}}}{\beta^{2} E} .
$$

To provide a first order estimation of the effect of space charge force on particle motion, we carry out linear approximation to the $\mathrm{rf}$ and space charge force on particle motion. Using a Gaussian bunch distribution with $\rho=$ $\left(N_{\mathrm{B}} / \sqrt{2 \pi} \sigma_{\tau}\right) \exp \left(-\left[\tau^{2} / 2 \sigma_{\tau}^{2}\right]\right)$, we find

$$
\Delta U_{\text {spch }}=+\frac{N_{\mathrm{B}} e^{2}\left|Z_{\mathrm{spch}}^{\|} / n\right|}{\sqrt{2 \pi} \omega_{0} \sigma_{\tau}^{2}} \frac{\tau}{\sigma_{\tau}} \exp \left\{-\frac{\tau^{2}}{2 \sigma_{\tau}^{2}}\right\} .
$$

For an order of estimation, we use $N_{\mathrm{B}}=1 \times 10^{9}$ particles $\left(I_{\text {avg }} \approx 315 \mu \mathrm{A}\right), \sigma_{\tau} \approx 15.0 \mathrm{~ns}$ with an $\mathrm{rf}$ voltage of $250 \mathrm{~V}$. The resulting space charge energy gain is $+23.2 e^{-\tau^{2} / 2 \sigma_{\tau}^{2}}\left(\tau / \sigma_{\tau}\right) \mathrm{eV}$. This is to be compared with the linear $\mathrm{rf}$ restoring force $e V \approx-e V_{\mathrm{rf}} \sin h \omega_{0} \tau \approx$ $-232\left(\tau / \sigma_{\tau}\right) \mathrm{eV}$. Thus the actual space charge force is about $10 \%$ of that of the $\mathrm{rf}$ focusing force.

Since the ratio of the space charge force to the rf force is proportional to $1 /\left(V_{\mathrm{rf}} \sigma_{\tau}^{3}\right) \sim V_{\mathrm{rf}}^{-1 / 4}$ when the bunch matches the bucket, the net effect of space charge force increases as the voltage is decreased. In our earlier example, the space charge force amounts to about $22 \%$ of that of the rf focusing force at $V_{\mathrm{rf}}=10 \mathrm{~V}$ and $27 \%$ at $V_{\mathrm{rf}}=5 \mathrm{~V}$. This large space-charge force does distort the rf potential well significantly. However, it is not large enough to totally cancel the rf focusing. In other words, there is still an rf bucket, although it has been distorted significantly. On the other hand, the rf voltage was brought down adiabatically, so that the bunch will always stay inside and fit the space-charge distorted bucket. Any loss of particles is a result of the possible nonadiabaticity of the rf maneuvering or microwave instability which we will discuss in the next subsection.

During the nonadiabatic bunch rotation stage, when the rf voltage is raised to a very high value, the bunch starts to rotate to the upright position for attaining a short bunch, the space charge force can become as important as that of low voltage situation, because the $V_{\mathrm{rf}}^{-1 / 4}$ rule does not apply anymore. However, this occurs only for a small fraction of the rotation and affects mostly particles near the center of the bunch. The potential well distortion gives rise to a smaller effective synchrotron tune near the center of the bunch. This may help to compensate the smaller synchrotron tune for large amplitude particles. We conclude that the potential well distortion should not be a critical factor in our experiment, and this is confirmed by numerical simulations.

\section{B. Microwave instabilities}

The situation most dangerous to microwave instability is when the rf is lowered adiabatically down to a few volts. Take the situation of $N_{\mathrm{B}}=1 \times 10^{9}$ protons in the bunch having an rms length of $\sigma_{\tau}=15$ ns filling the momentum aperture of the bucket at rf voltage $5 \mathrm{~V}$. The peak current is $I_{\text {peak }}=e N_{\mathrm{B}} / \sqrt{2 \pi} \sigma_{\tau}=4.26 \mathrm{~mA}$. The synchrotron tune is $\nu_{s}=8.23 \times 10^{-5}$. The half bucket height is found to be $\hat{\delta}=2 \nu_{s} /(h|\eta|)=5.23 \times 10^{-5}$. The Keil-Schnell criterion for stability of a Gaussian bunch is [7]

$$
\left|\frac{Z}{n}\right|<\frac{2 \pi \beta^{2} E|\eta| \sigma_{\delta}^{2}}{e I_{\text {peak }}}=156 \Omega,
$$

where we have used $\hat{\delta}=\sqrt{6} \sigma_{\delta}$. This limit is roughly 6.5 times less than the space charge impedance of the cooler ring. Simulations show that significant microwave instability and beam loss will occur if the space-charge impedance per harmonic $Z / n$ of the ring is around or larger than $1000 \Omega$. The growth is very fast and evolves fully in less than $0.5 \mathrm{~ms}$, which is less than $1 / 10$ of a synchrotron period. Since the rf bucket was mostly filled at $V_{\mathrm{rf}}=5 \mathrm{~V}$, any growth due to microwave instability will leave particles outside the bucket resulting to beam loss. Actually, we did see beam loss when $V_{\text {rf }}$ was lowered adiabatically to 10 or $5 \mathrm{~V}$. However, when we set $|Z / n|_{\text {spch }} \leq 1000 \Omega$ for the ring in the simulations, the effect of microwave instability is rather mild and becomes insignificant. This agrees with the well-known experience that the threshold of microwave instability below transition energy for a space charge dominated ring is many times above the Keil-Schnell limit [8].

\section{CONCLUSION}

At the IUCF cooler, the measured bunch compression ratios by the rf voltage and rf phase manipulation methods are compared with theory and numerical simulations. Naturally, the compression ratio depends on the initial rms bunch length and the highest attainable compression ratio is limited by the nonlinearities in the rf potential.

We find that the ultimate bunch compression ratio for the rf voltage manipulation is equal to the ratio of the maximum bucket area to the bunch area; i.e. $r_{c, \text { max }}^{v}=\mathcal{A}_{\mathrm{B}, \text { max }} / \mathcal{A}_{\text {bunch }}$. In the normalized synchrotron 
phase-space coordinates, the bucket area is 16 , and the bunch area is $6 \pi \sigma_{\phi, i}^{2}$, where we choose a factor of 6 for $95 \%$ of the beam, and $\sigma_{\phi, i}$ is the initial rms rf phase spread of the beam at the maximum rf voltage. Thus we find $r_{c, \text { max }}^{v} \approx 8 / 3 \pi \sigma_{\phi, i}^{2}$. In reality, one may be able to achieve $70 \%-80 \%$ of the maximum value because of nonlinearity in synchrotron motion.

We found that, on the average, a minimum of five synchrotron oscillation periods were required in order to guarantee adiabaticity during the bunch compression stage. The time required in the nonadiabatic voltage jump depends on the $Q$ of the rf system. Furthermore, during the rf voltage jump, beam loading can be an important issue that can induce dipole mode oscillation. In actual application, dipole mode oscillation is not important because the beam is kicked out at $1 / 4$ of the synchrotron motion.

The ultimate bunch compression ratio in the rf phase shift method is given by $r_{c, \text { max }}^{p}=\sqrt{2} / \sqrt{3} \sigma_{\phi, i}$. For the rf phase shift method, the time $t_{\text {UFP }}$ at the UFP during the bunch stretching stage is relatively short [see Eq. (22)]. The time for the rf phase jump depends on the $Q$ value of the rf system; generally, it is short compared with the synchrotron oscillation period. Since the peak current during the rf phase jump is not changed, the effect of beam loading will be minimized.

An advantage for the rf phase shift method is to avoid the nonlinearity in the rf potential by making the last stage of bunch rotation in the transfer line. At a desired compression ratio, the beam is kicked onto the transport line where a properly designed transport line with suitable $R_{56}$ transport matrix element can function as a buncher. Such a method will also work for bunch compression in electron storage rings, such as the damping ring for linear colliders. Since the synchrotron period is usually shorter than the damping time, the electron beam during the bunch compression stage at the UFP behaves like a proton bunch. The final stage of bunch rotation is carried out in the transfer line.

In order to provide a fair comparison of these two bunch compression methods, the initial bunch length should have the same initial voltage, i.e., $V_{2}$. In the rf voltage jump method, the rf voltage should be adiabatically decreased from $V_{2}$ to $V_{1}$, and nonadiabatically jumped from $V_{1}$ to $V_{2}$. The actual compression factor should be

$$
\tilde{r}_{c, \text { max }}^{v}=\left(\frac{V_{2}}{V_{1}}\right)^{1 / 4}=\left(\frac{\mathcal{A}_{\mathrm{B}, \max }}{\mathcal{A}_{\text {bunch }}}\right)^{1 / 2} \approx \frac{\sqrt{8}}{\sqrt{3 \pi} \sigma_{\phi, i}} .
$$

Comparing Eq. (28) with Eq. (21), we find that the voltage jump method is slightly more favorable. However, the rf voltage manipulation is limited by nonlinear synchrotron motion during the bunch rotation. The rf phase manipulation method can avoid nonlinear synchrotron motion by employing the transfer line for the final bunch rotation.

Finally, we also studied the space charge effect on bunch rotation. We found that the potential well distortion may help the bunch rotation in linearizing the synchrotron motion. However, the effect at the IUCF cooler is small. Since our experiments were carried out below transition energy, the microwave instability was not important.

We wish to acknowledge support from the U.S. Department of Energy Grant No. DE-FG02-92ER40747 and the National Science Foundation Grant No. PHY-9876773.

[1] S. Y. Lee et al., Phys. Rev. Lett. 67, 3768 (1991); D. D. Caussyn et al., Phys. Rev. A 46, 7942 (1992); D. D. Caussyn et al., Phys. Rev. Lett. 73, 2696 (1994); M. Ellison et al., Phys. Rev. E 50, 4051 (1994); D. D. Caussyn et al., Phys. Rev. E 51, 4947 (1995); S. Y. Lee et al., Phys. Rev. E 53, 1287 (1996).

[2] X. Kang, Ph.D. thesis, Indiana University, 1998 (unpublished).

[3] See, e.g., S. Y. Lee, Accelerator Physics (World Scientific, Singapore, 1999).

[4] M. Ellison et al., Phys. Rev. Lett. 70, 591 (1993); M. Syphers et al., Phys. Rev. Lett. 71, 719 (1993); D. Li et al., Phys. Rev. E 48, R1638 (1993); H. Huang et al., Phys. Rev. E 48, 4678 (1993).

[5] M. Bai et al., Phys. Rev. E 55, 3493 (1997).

[6] The ratio of bunch lengths derived from FWHM and rms analyses is about 2.95 instead of 2.355 for a Gaussian bunch. This may arise from the fact that the rms value of a Gaussian bunch is very sensitive to the background cut in our experimental data.

[7] S. Krinsky and J. M. Wang, Part. Accel. 17, 109 (1985).

[8] J. Bosser, C. Carli, M. Chanel, N. Madsen, S. Maury, D. Möhl, and G. Tranquille, Nucl. Instrum. Methods Phys. Res., Sect. A 441, 1 (2000). 